\title{
Comparison of effectiveness of parenteral lincomycin/spectinomycin combination and dexpanthenol application in goat kids with contagious ecthyma
}

\author{
SEVIM KASAP, ETHEM MUTLU TEMIZEL, ADIL ÖMER KARAKUŞ, OĞUZHAN AVCI*, \\ ESRA BÜYÜKCANGAZ**, SEZGIN SENTURK, FATIH KAVUKÇU, ORHAN YAPICI*

\begin{abstract}
Department of Internal Medicine, Faculty of Veterinary Medicine, Uludag University, Bursa, Turkey
*Department of Virology, Faculty of Veterinary Medicine, Selcuk University, Konya, Turkey

**Department of Microbiology, Faculty of Veterinary Medicine, Uludag University, Bursa, Turkey
\end{abstract}

\section{Kasap S., Temizel E. M., Karakuş A. O., Avcı O., Büyükcangaz E., Senturk S., Kavukçu F., Yapıcı O. Comparison of effectiveness of parenteral lincomycin/spectinomycin combination and dexpanthenol application in goat kids with contagious ecthyma}

\section{Summary}

Contagious ecthyma (CE) is a highly contagious viral skin disease that is typically self-limited. Treatment options include topical antiseptics, such as $\mathrm{KMNO}_{4}$, local antibiotics and systemic antibiotics to prevent secondary skin infections. The aim of this study was to compare the effectiveness of the lincomycin/spectinomycin combination and dexpanthenol (Dxp) in goat kids with CE. The study was conducted at a Saanen dairy goat farm in Bursa, Turkey. The owner of the goat herd inquired at the veterinary hospital about the appearance of granulomatous lesions on the muzzles of goat kids. In this study, 24 goat kids (1-month-olds) were used. All animals were subjected to the same conditions. Blood and papule samples were taken from the animals' lips, muzzle and buccal mucosa for virological analysis. Swab samples were taken from the lesions for culture and antibiogram. The animals were divided into three groups. Lesions were clinically scored at days 1,7 and 15 according to a modified previously used scoring system. Goat kids were equally grouped on the basis of lesions on the buccal mucosa, lips and muzzle. The animals in group A received $15 \mathrm{mg} / \mathrm{kg}$ lincomycin/ spectinomycin combination (Lypectin ${ }^{\circledR}$, Vilsan) intramuscularly for 3 consecutive days, group B received $20 \mathrm{mg} / \mathrm{kg}$ Dxp (Bepanthen ${ }^{\circledR}$ amp, Bayer, Germany) intramuscularly for 3 consecutive days, and group $\mathrm{C}$ received $0.9 \% \mathrm{NaCl}(2 \mathrm{ml})$, the control treatment. Clinical recoveries were almost equal in all groups, but by day 14 , group A showed better recovery than group $B$ and group $C$. Both study groups also showed better results than the control group for all days. In conclusion, we believe that the lincomycin/spectinomycin combination or dexpanthenol can be useful in the supplementary treatment of CE in goat kids.

Keywords: goat kids, dexpanthenol, lincomycin/spectinomycin, therapy, healing

Contagious ecthyma (CE) is a highly contagious viral skin disease that occurs in many ruminants, especially sheep and goats (21). The disease is also known as "Orf", contagious pustular dermatitis, or "sore mouth," and is much more severe in goat kids than it is in lambs (18). The causative agent is the Orf virus, a member of the Parapoxvirus genus of the Poxviridae family. The disease is widespread all over the world, and its prevalence increases especially during late summer and fall (12). While morbidity can reach $100 \%$ in farms where the disease occurs for the first time, mortality is approximately $5-10 \%$ when complicated by secondary bacterial infections (4). Once introduced to a herd, the disease can be quite challenging to control and eradicate.

Transmission occurs primarily through direct contact with a clinically affected animal or through indirect contact with soil and scabs that contain the virus. In addition, infection may result from iatrogenic transmission through surgical procedures, ear tagging and handling $(10,15)$. The incubation period of the disease varies from 4 to 8 days and is characterised by an initial rise in temperature and the development of papules and pustules often at the oral commissures, lips and nose. These symptoms are followed by thick, tenacious scabs 
covering a raised area of ulceration, granulation and inflammation (1). The diagnosis can be made by clinical examination of the lesions, but histopathology, PCR and ELISA methods are also used.

Because CE is self-limited and often heals spontaneously, treatment is often overlooked. Treatment options include topical antiseptics, such as $\mathrm{KMNO}_{4}$, boric acid (19), local antibiotics, and systemic antibiotics, such as lincosamides and macrolides, to prevent secondary skin infections. Goat kids with $\mathrm{CE}$ are more vulnerable to secondary infections caused by staphylococci, streptococci, Dermatophilus congolensis and Fusobacterium necrophorum, which can increase the severity of the disease (15). Lincomycin belongs to the lincosamide group of antibiotics and is widely used to treat bacterial skin infections caused by Streptococcus and most Staphylococcus types (16). Lincosamide antibiotics penetrates more effectively to the skin and bones, as well as most tissues. Furthermore, lincomycin also has immunomodulating effects (24).

Dexpanthenol (Dxp) is a biologically active alcohol-analogue of pantothenic acid (PA) and it is converted into PA inside the cell. PA is incorporated into the structure of coenzyme A, stimulates epithelization, and exerts anti-inflammatory effects (8). Dxp, a precursor of pantothenic acid (Vitamin $\mathrm{B}_{5}$ ), is used in veterinary medicine for intestinal atony or distension, postoperative paralytic ileus and equine colic to activate smooth muscle function. Because Dxp is rapidly converted into PA upon uptake, it is also essential for normal epithelial function. In both human and veterinary medicine, Dxp is used as a topical application to expedite epithelization in wound healing. Its dermatological effect occurs through fibroblast proliferation. Moreover, anti-inflammatory effects have also been reported (23).

The aim of this study was to compare the effectiveness of lincomycin/spectinomycin combination and Dxp in goat kids with CE.

\section{Material and methods}

Animals. This study was conducted for 2 weeks at a Saanen dairy goat farm in Bursa, Turkey. The study design was a blinded randomized controlled experiment. Goat kids were housed in the same barn at $20-24^{\circ} \mathrm{C}$, fed a standard commercial lamb start feed ( $200 \mathrm{~g} /$ day) and given water $\mathrm{ad}$ libitum.

Inclusion criteria and study design. The study was conducted on 24 Saanen goat kids diagnosed with different degrees of CE. All selected animals showed characteristic clinical signs of CE (granulomatous lesions around the nose, lips, and eyes) and were aged between 2 and 3 weeks. The animals were divided into 3 different treatment groups. One goat kid from each group was randomly selected for serological and virological examination. After confirmation of the serological and virological analysis, the kids were distributed to groups by age, sex and weight.
Tab. 1. Scab formation score Scab formation score* (no. of goats kids)

\begin{tabular}{c|c|r}
3 & 4 & formation \\
\hline $4(4 \times 3)$ & $2(2 \times 4)$ & 24
\end{tabular}

\begin{tabular}{l|l|l|l|l|l}
0 & $0(0 \times 1)$ & $2(2 \times 2)$ & $4(4 \times 3)$ & $2(2 \times 4)$ & 24
\end{tabular}

\begin{tabular}{l|l|l|l|l|r|}
3 & $1(1 \times 1)$ & $4(4 \times 2)$ & $2(2 \times 3)$ & $1(1 \times 4)$ & 19
\end{tabular}

\begin{tabular}{l|l|l|l|l|r}
7 & $4(4 \times 1)$ & $3(3 \times 2)$ & $0(0 \times 3)$ & $1(1 \times 4)$ & 14
\end{tabular}

\begin{tabular}{ll|l|l|l|l}
14 & $8(8 \times 1)$ & $0(0 \times 2)$ & $0(0 \times 3)$ & $0(0 \times 4)$ & $8^{\mathrm{a}}$
\end{tabular}

$\begin{array}{llllll}0 & 0(0 \times 1) & 3(3 \times 2) & 3(3 \times 3) & 2(2 \times 4) & 23\end{array}$

\begin{tabular}{ll|l|l|l|l}
3 & $2(2 \times 1)$ & $4(4 \times 2)$ & $1(1 \times 3)$ & $1(1 \times 4)$ & 17
\end{tabular}

\begin{tabular}{ll|l|l|l|l}
7 & $4(4 \times 1)$ & $3(3 \times 2)$ & $1(1 \times 3)$ & $0(0 \times 4)$ & 13
\end{tabular}

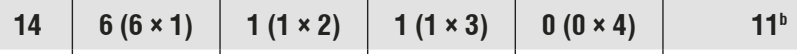

\begin{tabular}{llllll|l}
0 & $0(0 \times 1)$ & $2(2 \times 2)$ & $4(4 \times 3)$ & $2(2 \times 4)$ & 24
\end{tabular}

\begin{tabular}{ll|l|l|l|l}
3 & $0(0 \times 1)$ & $4(4 \times 2)$ & $3(3 \times 3)$ & $1(1 \times 4)$ & 21
\end{tabular}

\begin{tabular}{ll|l|l|l|l}
7 & $2(2 \times 1)$ & $4(4 \times 2)$ & $2(2 \times 3)$ & $0(0 \times 4)$ & 16
\end{tabular}

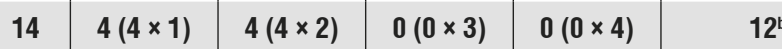

24
19
14
$8^{\mathrm{a}}$
23
17
13
$11^{\mathrm{b}}$
24
21
16
$12^{\mathrm{b}}$

s: 1 - no scab, 2 - mild scab, 3 - moderate scab, 4 - severe scab; ** group A - lincomycin/spectinomycin, group B - dexpanthenol, group $\mathrm{C}$ - control; $\mathrm{a}, \mathrm{b}$ - indicate statistically significant differences between

Lesions were scored at days 1, 3, 7 and 15 (Tab. 1) using a system based on a study by Musser et al. (13). Scab formation was scored by visual classification on a scale of 1 to 4 as follows: 1 - no scab, 2 - mild scab (two scabs on one side of the mouth), 3 - moderate scab (two scabs on both sides of the mouth), and 4 - severe scab (more than two scabs on both sides of the mouth). The same person assessed lesion scoring for all animals; lesion scores were verified by photographs. Each group included animals with equally severe baseline scores.

For our study, goat kids were divided into three groups. Each group consisted of 8 goat kids that clearly showed CE lesions on the buccal mucosa, lips and muzzle to varying degrees. Animals in group A received $15 \mathrm{mg} / \mathrm{kg}$ lincomycin/ spectinomycin combination (Lypectin ${ }^{\circledR}$, Vilsan) intramuscularly for 3 consecutive days, group B received $20 \mathrm{mg} / \mathrm{kg}$ Dxp (Bepanthen ${ }^{\circledR}$, Bayer, Germany) intramuscularly for 3 consecutive days, and group $\mathrm{C}$ received $2 \mathrm{ml} 0.9 \% \mathrm{NaCl}$ subcutaneously as the control treatment.

Sample collection and microbiological/virological analysis. Blood samples were collected from the animals, as well as scab lesion samples from the animals' lips, muzzle and buccal mucosa for diagnosis and isolation of the virus. All samples were obtained after centrifugation and stored at $-20^{\circ} \mathrm{C}$ until analysis. Blood samples were collected from the kids on day 1 (baseline) of this study. Swab samples were taken from the lesions for microbiological analysis and antibiogram.

Bacteriological analysis. All freshly collected lesion swabs were examined according to a routine diagnostic procedure by inoculating $7 \%$ defibrinated sheep blood with Columbia agar (COS 43041; BioMérieux ${ }^{\circledR}$, France), MacConkey’s agar $\left(\mathrm{CM}_{115}\right.$; Oxoid $\left.{ }^{\circledR}\right)$ and Levine Eosin Methylene Blue Agar $\left(\mathrm{CM}_{0069 \mathrm{~B}}\right.$; Oxoid $\left.{ }^{\circledR}\right)$. Petri dishes were incubated in a humidified atmosphere at $37^{\circ} \mathrm{C}$ for 24 hours. The swabs were then streaked onto Baired Parker Agar (Oxoid ${ }^{\circledR}$ CM 961) containing 5\% Egg Yolk Tellurite Emulsion (Oxoid ${ }^{\circledR}-$ SR0054C) and incubated for $48 \mathrm{~h}$ under aerobic conditions. One greyblack coloured Staphylococus spp. suspected colony was streaked onto Columbia Agar (Biomerioux $\left.{ }^{\circledR}-1001499590\right)$ 
to obtain pure isolate for use in identification. API-Staph ${ }^{\circledR}$ (Biomerioux $^{\circledR}$, Lyon, France) commercial identification panel was used for classifying candidate Staphylococcus isolates according to the manufacturer's guidelines. The results were evaluated by the API-Web ${ }^{\circledR}$ system.

Antimicrobial susceptibility testing. Kirby Bauer Disk Diffusion Technique was applied to all isolates according to EUCAST Disk Diffusion Method for Antimicrobial Susceptibility Testing - Version 6 (Jan 2017). Fourteen different antimicrobials were used for antimicrobial susceptibility testing of clinical isolates: enrofloxacine (ENR - Oxoid CT0639B), vancomycin (VA - Oxoid CT0058B), penicillin G (P-Oxoid CT0043B), cefoxitin (FOX - Oxoid CT0119B), mupirocin (MUP - Oxoid CT1826B), tetracycline (TE Oxoid CT0054B), lincomycin/spectinomycin (LS - Oxoid CT1758B), clindamycin (DA - Oxoid CT0064B), ampicillin/ sulbactam (SAM - Oxoid CT0520B), lincomycin/neomycin (LN - Oxoid CT1757B), amikacin (AK - Oxoid CT0107B), amoxycillin/clavulanic Acid (AMC - Oxoid CT0223B), sulphamethoxazole/trimethoprim (SXT - Oxoid CT0052B), gamithromycin (GAM - $15 \mu 1$-Mast Diagnostics). The zone diameters were calculated, and the results were evaluated according to "The European Committee on Antimicrobial Susceptibility Testing. Breakpoint tables for interpretation of MICs and zone diameters. Version 7.1" (valid from 2017-03-10).

Virological analysis. Scab lesion samples were homogenized (10\% w/v in PBS) with a Tissue Ruptor (Qiagen, Germany) in UltraPure DNase/RNase-Free Distilled Water and inoculated onto Madine Darby Bovine Kidney (MDBK) permanent cell lines. DNA was extracted from infected MDBK cells using the QIAamp cador Pathogen Mini Kit (Qiagen, Germany) according to the manufacturer's instructions. A set of primers were designed based on the Orf virus B2L gene (7). PCR mix included $0.2 \mu \mathrm{M}$ PP1 and PP4 primers, $200 \mu \mathrm{M}$ dNTPs, $1.5 \mathrm{mM} \mathrm{MgCl}$, $1 \mathrm{U}$ Taq DNA polimerase enzyme and $5 \mu \mathrm{DNA}$ sample. All amplifications were performed as follows: $94^{\circ} \mathrm{C}$ for $2 \mathrm{~min}, 35$ cycles of $94^{\circ} \mathrm{C}$ for 35 seconds, $60^{\circ} \mathrm{C}$ for 35 seconds and $72^{\circ} \mathrm{C}$ for 45 seconds, annealing at $72^{\circ} \mathrm{C}$ for $5 \mathrm{~min}$ on an MJ PTC-200 thermal cycler. PCR products were analyzed on $1.5 \%$ agarose gel in $1 \mathrm{XTBE}$ buffer. 594 bp products were considered as positives. Electrophoresis was carried out at $1 \mathrm{~V} / \mathrm{cm}$ for $16 \mathrm{~h}$ at room temperature.
Statistical analysis. Data were analyzed using the oneway repeated measures analysis of variance. Tukey tests was performed as post hoc test. Student's t-tests were used to test the significance of differences between the study and control groups. A p-value $<0.05$ was considered significant. All statistical analyses were performed using the Sigma Stat 3.1 for Windows statistical package (Systat Software, Point Richmond, CA).

\section{Results and discussion}

Except for the mouth lesions, clinical examination of the animals revealed no problems throughout the study. Clinical parameters were within reference limits. In group A $(n=8)$, lincomycin/spectinomycin induced the healing of scabs at day 14 . Group B $(n=8)$ showed better healing of the scabs than group A (Tab. 1) at days 3 and 7 . Group C $(n=8)$, the control group that received only $\mathrm{NaCl}$, had the worst score of all three groups, indicating poor healing of the scabs at days 3, 7 and 14 (Tab. 1).

Bacteriological analysis and antimicrobial susceptibility testing. Staphylococcus aureus was detected in all swabs. The antimicrobial susceptibility pattern of Staphylococcus aureus isolates is shown in Fig. 1. Isolates 409 and 400 had intermediate susceptibility to cefoxitin, and three isolates $(400,342,421)$ were resistant to tetracycline. Isolates 342 and 421 also had intermediate susceptibility to gamithromycin.

$\mathrm{CE}$ is one of the most common skin diseases of sheep and goats worldwide. The clinical signs of the disease are most characteristically observed in goat kids and lambs. $\mathrm{CE}$ is a self-limiting disease, and spontaneous recovery without treatment occurs within 3-4 weeks in susceptible species. However, the recovery period for CE can be affected by some variables, including nutritional and passive transfer status, season type, and the presence of a secondary bacterial infection, such as staphylococci, alpha haemolytic streptococci or Corynebacterium (2, $15)$. These variables may increase the mortality rate of the affected animals (13). In this study, goat kids had granulomatous lesions around the mouth, eyes and nose. No goat kids in the herd died during the outbreak. Our observations determined that the goat kids recov-

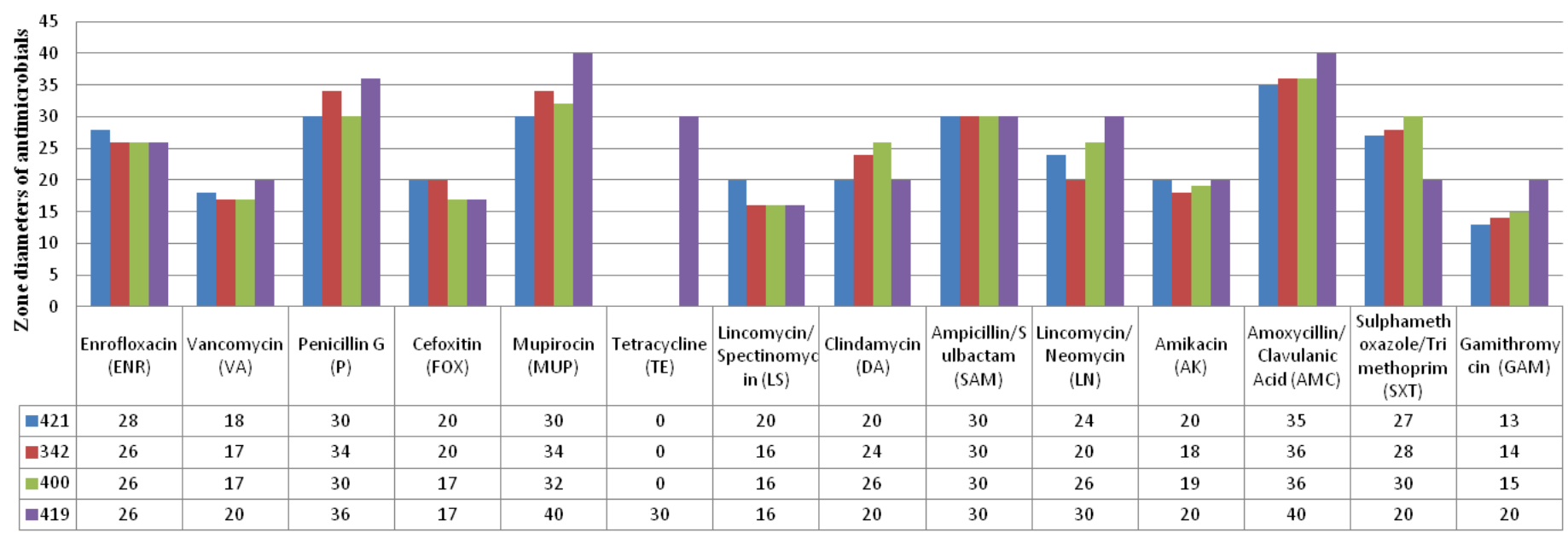

Fig. 1. Antimicrobial susceptibility pattern of Staphylococcus aureus isolates from ecthyma cases in goat kids by Kirby Bauer Disc Diffusion Method 
ered spontaneously within 2-4 weeks. In cases of CE, different therapeutic alternatives should be evaluated, considering deaths due to different variables. Because of the risk of disease transmission and death, treatments for $\mathrm{CE}$ should be evaluated.

In dermatologic diseases, determination of the location and size of lesions is essential for evaluating the severity of the disease and the recovery time. For this reason, a scoring system has been used in many studies $(13,21)$. In the present study, lesions were scored by visual examination of scab formation on a scale of 1 to 4 based on a study by Musser et al. (13): 1 - no scab, 2 - mild scab, 3 - moderate scab, and 4 - severe scab. As previously described, this scoring system is easy to use and sensitive enough to detect clinical responses to therapy. The severity of CE lesions may depend on the species, breed, age, crowded pen conditions and nutritional status $(13,15,18)$. Therefore, this study used animals of the same age group. In addition, all animals were enclosed in a separated area under the same barn conditions.

Although CE does not cause death directly, the disease can be fatal if its lesions prevent lambs and kids from suckling, as these animals can be more sensitive to secondary bacterial or fungal infections (5). The morbidity of the disease may reach $100 \%$, and mortality, due to secondary bacterial infections, may reach $15 \%$ (4). Therefore, antibacterial treatment may be necessary in cases of CE complicated by secondary bacterial infection $(14,15)$. Lincosamides are among the first-choice bacteriostatic antibiotics used in veterinary dermatology. They are well absorbed if administered orally and penetrate well into the infected skin. Their spectrum of action comprises bacteria commonly associated with skin infections, including Staphylococci spp. (17). Lincomycin/spectinomycin has previously been reported to be effective in the treatment of ovine footrot (25). This combination is also effective in skin infections caused by Dermatophilosis spp. seen in sheep (9). In addition, Jordan and Venning (9) reported a 50\% success rate in resolving lesions in sheep given lincomycin/spectinomycin. In the present study, the lincomycin/spectinomycin treatment (group A) was marked by a rapid recovery after day 3 of treatment compared to group C. In addition, the recovery of group A was significantly different than that of groups B and C at day 14 of treatment $(\mathrm{p}<0.05)$. We found that Staphylococcus aureus showed mild sensitivity to lincomycin/spectinomycin in the antibiogram (Fig. 1). In clinical observation, lincomycin/spectinomycin had a partial effect compared to the control group.

Dxp is used topically as a healing agent in many skin diseases $(11,20)$. Previous studies have suggested that topical formulations containing Dxp were effective in treatment and prevention of dermatological lesions $(3,6$, 22). However, to our knowledge, no published study has shown the effect of Dxp on dermatologic lesions with parenteral application. In the present study, the animals that received Dxp parenterally (group B) healed faster than animals that received the control treatment (group C). This study showed that Dxp may be used topically or parenterally as a support therapy in the treatment of $\mathrm{CE}$ because of its positive effect on the clinical score and healing.

In conclusion, our results show that lincomycin/spectinomycin can effectively heal CE scabs in goat kids. In addition, parenteral Dxp can be used as an alternative therapy for animals with CE.

\section{References}

1. Chan K. W., Lin J. W., Lee S. H., Liao C. J., Tsa M. C., Hsu W. L., Wong M. L., Shih H. C.: Identification and phylogenetic analysis of orf virus from goats in Taiwan. Virus Genes 2007, 35, 705-712.

2. De La Concha-Bermejillo A., Guo J., Zhang Z., Waldron D.: Severe persistent orf in young goats. J. Vet. Diagn. Invest. 2003, 15, 423-431.

3. Ebner F., Heller A., Rippke F., Tausch I.: Topical use of dexpanthenol in skin disorders. Am. J. Clin. Dermatol. 2002, 3, 427-433.

4. Gumbrell R. C., Mcgregor D. A.: Outbreak of severe fatal orf in lambs. Vet. Rec. 1997, 141, 150-151.

5. Haig M., McInnes C. J.: Immunity and counter-immunity during infection with the parapoxvirus orf virus. Virus Res. 2002, 88, 3-16.

6. Heise R., Skazik C., Marquardt Y., Czaja K., Sebastian K., Kurschat P., Gan I., Denecke B., Ekanayake-Bohlig S., Wilhelm K. P., Merk H. F., Baron J. M: Dexpanthenol modulates gene expression in skin wound healing in vivo. Skin Pharmacol. Physiol. 2012, 25, 241-248.

7. Inoshima Y., Morooka A., Sentsui H.: Detection and diagnosis of parapoxvirus by the polymerase chain reaction. J. Virol. Methods 2000, 84, 201-208.

8. Jessop C. E., Bulleid N. J.: Glutathione directly reduces an oxidoreductase in the endoplasmic reticulum of mammalian cells. J. Biol. Chem. 2004, 279, 55341-55347.

9. Jordan D., Vening C.: Treatment of ovine dermatophilosis with long acting oxytetracycline or a lincomycin spectinomycin combination. Aust. Vet. J. 1995, 72, 234-236

10. Kumar R., Trivedi R. N., Bhatt P., Khan S. U. H., Khurana S. K., Tiwari R., Karthik K., Malik Y. S., Dhama K., Chandra R.: Contagious pustular dermatitis (orf disease) - epidemiology, diagnosis, control and public health concerns. Adv. Anim. Vet. Sci. 2015, 3, 649-676.

11. Løkkevik E., Skovlund E., Reitan J. B.: Skin treatment with bepanthen cream versus no cream during radiotherapy - a randomized controlled trial. Acta Oncol. 1996, 35, 1021-1026.

12. McKeever D. J., Reid H. W.: Survival of orf virus under British winter conditions. Vet. 1986, 118, 613-614.

13. Musser J., Taylor C., Guo J., Tizard I., Walker J.: Development of a contagious ecthyma vaccine for goats. Am. J. Vet. Res. 2008, 69, 1366-1370.

14. Nandi S., Rao T. V. S., Malik P.: Sheep pox - a scourge to sheep industry in India. Ind. Farming 1999, 49, 29-31

15. Nand S., De U., Sumit C.: Current status of contagious ecthyma or orf disease in goat and sheep - A global perspective. Small Rumi. Res. 2011, 96, 73-82.

16. Noble $W$. C.: Topical and systemic antibiotics: is there a rationale? Semin Dermatol. 1990, 9, 250-254.

17. Noll C., Boothe D.: Macrolides and lincosamides. Vet. Dermatol. 1999, 10, 217-223.

18. Pugh D. C., Baird N.: Contagious Ecthyma, [in:] Sheep and Goat Medicine, vol 2, Saunders, Philadelphia 2012, p. 234

19. Rao B. T., Das H. J., Sharma R. D., Singh S. S.: Some observations on an outbreak of sheep pox in sheep in East Godavari District, Andhra Pradesh. Livest. Advis. 1994, 19, 3-6.

20. Schmuth M., Wimmer M. A., Hofer S., Sztankay A., Weinlich G., Linder D. M. Elias P. M., Fritsch P. O., Fritsch E.: Topical corticosteroid therapy for acute radiation dermatitis: a a prospective, randomized, double-blind study. Br. J. Dermatol. 2002, 146, 983-991.

21. Spyrou V., Valiakos G.: Orf virus infection in sheep or goats. Vet. Microbiol. 2015, 181, 178-182.

22. Stożkowska W., Piękoś R.: Investigation of some topical formulations containing dexpanthenol. Acta Pol. Pharm. 2004, 61, 433-437.

23. Toplu Y., Sapmaz E., Parlakpinar H., Kelles M., Kalcioglu M. T., Tanbek K., Kizilay A.: The effect of dexpanthenol on ototoxicity induced by cisplatin. Clin. Exp. Otorhinolaryngol. 2016, 9, 14-20.

24. Tufano M. A., Cipollaro de l'Ero G., Ianniello R., Baroni A., Galdiero F.: Antimicrobial agents induce monocytes to release IL-1 alpha, IL-6, and TNF, and induce lymphocytes to release IL-4 and TNF tau. Immunopharmacol. Immunotoxicol. 1992, 14, 769-782.

25. Venning C. M., Curtis M. A., Egerton J. R.: Treatment of virulent footrot with lincomycin and spectinomycin. Aust. Vet. J. 1990, 67, 258-260.

Corresponding author: Sevim Kasap, PhD, Department of Internal Medicine, Faculty of Veterinary Medicine, Uludag University, Bursa, Turkey; e-mail: cetins@uludag.edu.tr 